\title{
Produção especializada no SUS em capitais brasileiras com centros de especialidades odontológicas: uma análise descritiva
}

\author{
Specialized production in the unified health system in Brazilian capitals \\ with dental specialty centers: a descriptive analysis
}

\author{
Alissa Schmidt San Martin \\ Ketlen Conde** \\ Luane Morales ${ }^{* * *}$ \\ Marcos Britto Corrêa ${ }^{* * * *}$ \\ Marcus Cristian Muniz Conde ${ }^{* * * * *}$ \\ Luiz Alexandre Chisini ${ }^{* * * * *}$
}

\section{Resumo}

Centros de especialidades odontológicos (CEOs) são estabelecimentos de saúde de âmbito especializado que devem realizar uma quantidade mínima de procedimentos. Objetivos: descrever a produção odontológica especializada e reportar o cumprimento das metas nas capitais brasileiras com CEOs. Materiais e método: foi conduzido um estudo do tipo longitudinal retrospectivo, sendo realizada uma busca por CEOs cadastrados no Cadastro Nacional de Estabelecimentos de Saúde (CNES). A produção odontológica foi pesquisada no Sistema de Informações Ambulatoriais do Sistema Único de Saúde (SIASUS), de maio de 2015 a abril de 2016. Resultados: foram encontrados e considerados elegíveis para o presente estudo 59 CEOs, localizados em $19 \mathrm{ca}$ pitais brasileiras e no Distrito Federal, sendo $48 \%$ CEOs tipo II. Cerca de 730 mil procedimentos especializados foram realizados durante os 12 meses avaliados. Uma taxa de $86 \%$ das metas foi cumprida, sendo que cirurgia foi a área com maior cumprimento $(92 \%)$, seguida de periodontia $(89 \%)$ e endodontia $(76 \%)$. Uma das capitais apresentou apenas 33\% das metas cumpridas. Três capitais não atingiram nenhuma das metas estabelecidas em procedimentos de endodontia. Conclusão: foi observada uma grande variação no cumprimento das metas entre as capitais com CEOs. Enquanto algumas capitais apresentaram elevado cumprimento das metas, outras exibiram dados preocupantes, principalmente nos procedimentos de endodontia.

Palavras-chave: Avaliação dos serviços de saúde. Especialidades odontológicas. Saúde bucal. Saúde pública. Serviços de saúde bucal.

\section{Introdução}

A saúde pública no Brasil tem passado por importantes transformações nas últimas décadas ${ }^{1,2}$, principalmente devido à criação de políticas públicas de saúde, como o Programa Saúde da Família $(\mathrm{PSF})^{3}$. Esse programa tem como principais objetivos a reorganização e o fortalecimento da atenção básica por meio de princípios de equidade, universalidade e integralidade ${ }^{4}$. No ano de 2004 , a odontologia foi incorporada nestas novas ações de saúde com a criação da Política Nacional de Saúde Bucal (PNSB), conhecida como Brasil Sorridente ${ }^{4}$. Dessa forma, ocorreu uma inversão na atenção à saúde bucal, que era definida pela realização de procedimentos clínicos mutiladores e pela atuação preventiva individualizada $^{5}$. Um novo modelo, caracterizado por atuação coletiva e realização de procedimentos visando à manutenção da saúde, passou a ser incorporado como rotina ${ }^{2,5}$. De fato, ocorreu uma ampliação do acesso gratuito a tratamentos odontológicos com a qualificação de ações e serviços oferecidos e com a criação das equipes de saúde bucal (ESBs), que passaram a atuar conjuntamente na Estratégia de Saúde da Família (ESF) ${ }^{1,6}$.

Essa atuação conjunta elevou a produção odontológica na atenção básica em quase $50 \%$ nos municípios beneficiados com as equipes entre os anos de 1999 e $2011^{7}$. Assim, estima-se que cerca de $80 \%$

\footnotetext{
Acadêmica da Faculdade de Odontologia, Universidade Federal de Pelotas, Brasil.

Acadêmica da Faculdade de Odontologia, Universidade Federal de Pelotas, Brasil.

Acadêmica da Faculdade de Odontologia, Universidade Federal de Pelotas, Brasil.

Doutor em Odontologia. Professor do Departamento de Odontologia Restauradora, Universidade Federal de Pelotas, Pelotas, Rio Grande do Sul, Brasil. Doutor em Odontologia. Professor do Centro de Ciências Biológicas e da Saúde, Universidade do Vale do Taquari, Lajeado, Rio Grande do Sul, Brasil. Mestre em Dentística. Professor do Centro de Ciências Biológicas e da Saúde, Universidade do Vale do Taquari, Lajeado, Rio Grande do Sul, Brasil.
} 
das necessidades odontológicas dos usuários do Sistema Único de Saúde (SUS) possam ser sanadas na atenção básica ${ }^{1}$. Quando a complexidade dos procedimentos extrapola as possibilidades das unidades básicas de saúde (UBS), estes podem ser referenciados para os centros de especialidades odontológicas (CEOs) ou para os laboratórios regionais de prótese dentária (LRPDs) ${ }^{1}$. Na última década, além da incorporação da atenção secundária, que era praticamente inexistente, ocorreu uma expansão de $445 \%$ das ESBs. Por conseguinte, 2,1 milhões de próteses dentárias foram entregues nas quase 2 mil cidades que apresentavam LRPD; tornando o Brasil Sorridente um dos maiores programas de saúde bucal do mundo ${ }^{1}$. Apesar dos impressionantes números, o programa também apresenta inúmeros desafios ${ }^{5}$. Um recente estudo avaliou a produção odontológica em municípios com CEOs no sul do Brasil e observou que $26 \%$ dos municípios atingiram menos da metade das metas estabelecidas ${ }^{8}$.

Os CEOs são estabelecimentos de saúde de âmbito especializado que devem realizar uma quantidade mínima de procedimentos, variando de acordo com a modalidade de CEO. As especialidades que devem estar presentes no CEO são: periodontia clínica, endodontia, cirurgia oral menor dos tecidos moles e duros, diagnóstico bucal com ênfase na detecção de câncer bucal e procedimentos básicos realizados exclusivamente em pacientes com necessidades especiais ${ }^{9}$. A implantação dos CEOs é realizada pela iniciativa dos gestores, que podem ser municipais, estaduais ou ambos. Além disso, existem três tipos de CEO, os quais devem realizar um número mínimo de procedimentos por mês em cada uma das especialidades. Por sua vez, os procedimentos devem ser registrados pelos gestores no Sistema de Informações Ambulatoriais do SUS (SIASUS), que é utilizado como ferramenta para controle e monitoramento dos $\mathrm{CEOs}^{9}$. Tendo em vista que poucos estudos têm investigado a produção odontológica especializada em locais com CEOs, os objetivos do presente estudo foi descrever a produção odontológica especializada nas capitais brasileiras e no Distrito Federal com CEOs e analisar se a produção de média complexidade desses municípios condiz com a quantidade de CEOs presentes.

\section{Materiais e método}

Foi conduzido um estudo do tipo longitudinal retrospectivo, com uso de dados provenientes do SIASUS e do Cadastro Nacional de Estabelecimentos em Saúde (CNES), como descrito por Pires et al. ${ }^{8}$ (2015). A coleta de dados foi realizada durante o período de agosto a setembro de 2016.

O SIASUS é um banco de dados de domínio público que visa dar, de forma informatizada, transparência aos dados do SUS. Nesse sistema, é possível obter a quantidade de procedimentos odontológicos realizados em cada município brasileiro. O monitoramento dos procedimentos realizados pelos CEOs deve ser realizado por intermédio desse sistema, propiciando um maior controle por parte dos gestores e possibilitando o planejamento das políticas públicas. Além disso, todos os estabelecimentos de saúde, sejam de âmbito público ou privado, devem estar cadastrados no CNES. Assim, todos os CEOs devem estar cadastrados nesse sistema.

Dois autores treinados (ASSM e LM) realizaram a coleta das variáveis de forma independente. Inconsistências foram checadas por um terceiro e autor experiente (LAC). Inicialmente, foi realizada uma busca por CEOs cadastrados no CNES (cnes. datasus.gov.br) em todas as capitais brasileiras e no Distrito Federal. Após a identificação dos CEOs, foi averiguada sua classificação no próprio CNES, que também apresenta a modalidade de CEO. Quando a modalidade de CEO não estava presente, a categorização era feita de acordo com o número de cadeiras odontológicas presentes, uma vez que esse é um dos critérios de classificação dos CEOs. Assim, os CEOs que não apresentavam explicitamente sua modalidade foram classificados em:

a) CEO tipo I: 3 cadeiras odontológicas;

b) CEO tipo II: de 4 a 6 cadeiras odontológicas;

c) CEO tipo III: acima de 7 cadeiras odontológicas.

A produção odontológica nos municípios que apresentavam CEOs ativos foi pesquisada no SIASUS pelo site do banco de dados do Departamento de Informática do SUS (DATASUS). Os procedimentos consultados foram todos os de âmbito especializado descritos na Portaria 1.464, de 24 de junho de 2011, que define a produção odontológica mínima a ser realizada para cada tipo de $\mathrm{CEO}^{9}$. Procedimentos de atenção básica presentes nessa portaria não foram computados. Assim, os procedimentos realizados durante o período de maio de 2015 a abril de 2016 foram pesquisados diretamente no banco do DATASUS, item informações de saúde, subitem produção ambulatorial, por gestor - a partir de 2008. Os dados foram tabulados mês a mês, criando uma série histórica no programa Microsoft Excel, versão 2013 (Microsoft Corp.).

Os procedimentos foram agrupados de acordo com a Portaria Interministerial 1.464, de 24 de junho de 2011, seguindo três grupos: procedimentos de periodontia, procedimentos de endodontia e procedimentos de cirurgia oral menor ${ }^{9}$. $\mathrm{O}$ cumprimento das metas estipuladas foi analisado mês a mês, referente a cada grupo de procedimento, em cada um dos municípios. As metas foram de acordo com o tipo e o número de CEOs cadastrados para cada cidade durante o período avaliado. Assim, foram tabulados dicotomicamente numa planilha, entre os que atingiam e os que não atingiam as metas estabelecidas em cada um dos meses. Os dados foram analisados de maneira descritiva, estimando-se frequências absolutas e relativas das variáveis de interesse. 


\section{Resultados}

Foram encontrados e considerados elegíveis para o presente estudo $59 \mathrm{CEOs,}$, sendo localizados em 19 capitais brasileiras e no Distrito Federal. Quase a metade dos CEOs presentes eram do tipo II $-48 \%$ (28), enquanto que $25 \%$ (15) eram tipo I e
$27 \%$ (16) eram do tipo III. São Paulo foi a capital que mais apresentou CEOs, totalizando oito unidades, e a maioria (50\%) também foi do tipo II. Os demais CEOs distribuídos nas capitais brasileiras são apresentados na Tabela 1.

Tabela 1 - Capitais brasileiras com CEOs cadastrados no CNES* e respectivas quantidades de CEOs de acordo com suas modalidades

\begin{tabular}{|c|c|c|}
\hline Capitais & Tipo de CEO (quantidade) & Número total de CEOs \\
\hline Belo Horizonte & Tipo I (0); Tipo II (2); Tipo III (2) & 4 \\
\hline Boa Vista & Tipo I (0); Tipo II (2); Tipo III (0) & 2 \\
\hline Brasília & Tipo I (0); Tipo II (1); Tipo III (0) & 1 \\
\hline Campo Grande & Tipo I (0); Tipo II (3); Tipo III (1) & 4 \\
\hline Curitiba & Tipo I (0); Tipo II (1); Tipo III (2) & 3 \\
\hline Florianópolis & Tipo I (0); Tipo II (1); Tipo III (0) & 1 \\
\hline Fortaleza & Tipo I (2); Tipo II (3); Tipo III (0) & 5 \\
\hline Goiânia & Tipo I (4); Tipo II (0); Tipo III (0) & 4 \\
\hline João Pessoa & Tipo I (0); Tipo II (2); Tipo III (0) & 2 \\
\hline Macapá & Tipo I (0); Tipo II (0); Tipo III (2) & 2 \\
\hline Maceió & Tipo I (0); Tipo II (1); Tipo III (0) & 1 \\
\hline Manaus & Tipo I (0); Tipo II (2); Tipo III (3) & 5 \\
\hline Palmas & Tipo I (0); Tipo II (0); Tipo III (1) & 1 \\
\hline Porto Alegre & Tipo I (4); Tipo II (0); Tipo III (0) & 4 \\
\hline Rio Branco & Tipo I (1); Tipo II (0); Tipo III (0) & 1 \\
\hline Salvador & Tipo I (1); Tipo II (2); Tipo III (3) & 6 \\
\hline São Luís & Tipo I (1); Tipo II (2); Tipo III (0) & 3 \\
\hline São Paulo & Tipo I (2); Tipo II (4); Tipo III (2) & 8 \\
\hline Tersesina & Tipo I (0); Tipo II (1); Tipo III (0) & 1 \\
\hline Vitória & Tipo I (0); Tipo II (1); Tipo III (0) & 1 \\
\hline
\end{tabular}

${ }^{*}$ CEOs cadastrados no CNES até abril de 216.

Fonte: CNES.

Cerca de 730 mil procedimentos especializados foram realizados pelos municípios durante os 12 meses avaliados. Do total de procedimentos especializados realizados, cerca de 374 mil (51\%) foram procedimentos de periodontia, seguidos de 242 mil (33\%) de cirurgia oral menor e 113 mil $(15,5 \%)$ de endodontia. São Paulo foi a capital avaliada que mais realizou procedimentos especializados, execu- tando um montante de, aproximadamente, $159 \mathrm{mil}$ procedimentos. Em contrapartida, Rio Branco foi a capital avaliada que menos apresentou procedimentos especializados (cerca de 7 mil). De forma geral, todas as capitais avaliadas apresentaram uma considerável produção especializada nas respectivas áreas (Tabela 2 ).

Tabela 2 - Procedimentos odontológicos especializados* ${ }^{\star}$ registrados no SIASUS nas capitais brasileiras com CEOs

\begin{tabular}{|c|c|c|c|c|c|c|c|}
\hline & \multicolumn{2}{|c|}{ Periodontia } & \multicolumn{2}{|c|}{ Endodontia } & \multicolumn{2}{|c|}{ Cirurgia oral menor } & \multirow[t]{2}{*}{ Total } \\
\hline & Absoluto & $\%$ & Absoluto & $\%$ & Absoluto & $\%$ & \\
\hline Belo Horizonte & 13.196 & 44,34 & 9.108 & 30,60 & 7.460 & 25,06 & 29.764 \\
\hline Boa Vista & 1.496 & 15,66 & 1.472 & 15,41 & 6.582 & 68,92 & 9.550 \\
\hline Brasília & 13.439 & 40,33 & 3.322 & 9,97 & 16.564 & 49,70 & 33.325 \\
\hline Campo Grande & 19.095 & 47,42 & 10.992 & 27,30 & 10.184 & 25,29 & 40.271 \\
\hline Curitiba & 7.949 & 26,06 & 9.149 & 29,99 & 13.407 & 43,95 & 30.505 \\
\hline Florianópolis & 5.011 & 33,87 & 4.509 & 30,47 & 5.276 & 35,66 & 14.796 \\
\hline Fortaleza & 72.497 & 75,36 & 6.263 & 6,51 & 17.444 & 18,13 & 96.204 \\
\hline Goiânia & 13.678 & 54,21 & 4.048 & 16,04 & 7.507 & 29,75 & 25.233 \\
\hline João Pessoa & 3.670 & 18,56 & 9.020 & 45,61 & 7.087 & 35,83 & 19.777 \\
\hline Macapá & 6.379 & 62,55 & 999 & 9,80 & 2.821 & 27,66 & 10.199 \\
\hline Maceió & 1.280 & 14,68 & 2.478 & 28,42 & 4.960 & 56,89 & 8.718 \\
\hline Manaus & 65.057 & 67,01 & 11.991 & 12,35 & 20.035 & 20,64 & 97.083 \\
\hline Palmas & 10.287 & 72,11 & 519 & 3,64 & 3.459 & 24,25 & 14.265 \\
\hline Porto Alegre & 9.028 & 52,84 & 2.002 & 11,72 & 6.054 & 35,44 & 17.084 \\
\hline Rio Branco & 2.443 & 34,52 & 1.129 & 15,95 & 3.505 & 49,53 & 7.077 \\
\hline Salvador & 35.875 & 53,68 & 5.594 & 8,37 & 25.365 & 37,95 & 66.834 \\
\hline São Luís & 8.283 & 30,69 & 6.200 & 22,98 & 12.502 & 46,33 & 26.985 \\
\hline São Paulo & 78.903 & 49,67 & 15.684 & 9,87 & 64.262 & 40,45 & 158.849 \\
\hline Teresina & 3.008 & 19,06 & 7.667 & 48,59 & 5.105 & 32,35 & 15.780 \\
\hline Vitória & 4.102 & 48,35 & 1.144 & 13,48 & 3.238 & 38,17 & 8.484 \\
\hline Total & 374.676 & 51,27 & 113.290 & 15,50 & 242.817 & 33,23 & 730.783 \\
\hline
\end{tabular}

Fonte: SIASUS. 
Em relação ao cumprimento das metas, foi observado que $86 \%$ das metas de todas as capitais e do Distrito Federal foram cumpridas. As metas mais atingidas foram as de cirurgia oral menor $(92 \%)$, seguida de periodontia (89\%) e endodontia (76\%) (Tabela 3). Além disso, foi possível observar que 45\% das cidades que apresentavam CEOs atingiram $100 \%$ das metas estabelecidas. No entanto, uma das capitais (Macapá) apresentou apenas $33 \%$ das metas cumpridas durante o período, não atingindo nenhuma meta nos procedimentos de endodontia. De forma semelhante, foi observado que os procedi- mentos de endodontia foram os que apresentaram menores cumprimentos. Dentre os procedimentos endodônticos, três capitais (Macapá, Maceió e Palmas) não atingiram nenhuma das metas estabelecidas, nos doze meses avaliados, e duas capitais (Boa Vista e Salvador) atingiram apenas $33 \%$ das metas. Procedimentos da área de cirurgia oral menor foram os que apresentaram os maiores cumprimentos das metas (92\%), sendo que apenas em uma capital foi observado apenas $9 \%$ de cumprimento, enquanto que cerca de $70 \%$ das capitais atingiram $100 \%$ das metas.

Tabela 3 - Análise descritiva das metas atingidas das capitais brasileiras com CEOs, distribuídas por especialidade no período de março de 2015 a abril de 2016

\begin{tabular}{|c|c|c|c|c|}
\hline Municípios & Periodontia (\%) & Endodontia (\%) & Cirurgia oral menor (\%) & Total $\%$ \\
\hline Belo Horizonte & $67 \%$ & $100 \%$ & $75 \%$ & $81 \%$ \\
\hline Boa Vista & $41 \%$ & $33 \%$ & $75 \%$ & $74 \%$ \\
\hline Brasília & $100 \%$ & $100 \%$ & $100 \%$ & $100 \%$ \\
\hline Campo Grande & $100 \%$ & $100 \%$ & $100 \%$ & $100 \%$ \\
\hline Curitiba & $100 \%$ & $100 \%$ & $100 \%$ & $100 \%$ \\
\hline Florianópolis & $100 \%$ & $100 \%$ & $100 \%$ & $100 \%$ \\
\hline Fortaleza & $100 \%$ & $83 \%$ & $100 \%$ & $85 \%$ \\
\hline Goiânia & $100 \%$ & $100 \%$ & $100 \%$ & $100 \%$ \\
\hline João Pessoa & $50 \%$ & $83 \%$ & $91 \%$ & $75 \%$ \\
\hline Macapá & $91 \%$ & $0 \%$ & $9 \%$ & $33 \%$ \\
\hline Maceió & $59 \%$ & $0 \%$ & $100 \%$ & $53 \%$ \\
\hline Manaus & $100 \%$ & $100 \%$ & $100 \%$ & $100 \%$ \\
\hline Palmas & $100 \%$ & $0 \%$ & $100 \%$ & $67 \%$ \\
\hline Porto Alegre & $100 \%$ & $91 \%$ & $100 \%$ & $97 \%$ \\
\hline Rio Branco & $91 \%$ & $100 \%$ & $100 \%$ & $97 \%$ \\
\hline Salvador & $100 \%$ & $33 \%$ & $91 \%$ & $75 \%$ \\
\hline São Luís & $100 \%$ & $100 \%$ & $100 \%$ & $100 \%$ \\
\hline São Paulo & $100 \%$ & $100 \%$ & $100 \%$ & $100 \%$ \\
\hline Teresina & $83 \%$ & $100 \%$ & $91 \%$ & $91 \%$ \\
\hline Vitória & $100 \%$ & $100 \%$ & $100 \%$ & $100 \%$ \\
\hline Total & $89 \%$ & $76 \%$ & $92 \%$ & $86 \%$ \\
\hline
\end{tabular}

Fonte: SIASUS.

\section{Discussão}

O presente estudo observou uma expressiva produção de média complexidade realizada exclusivamente pelo SUS em 19 capitais brasileiras e no Distrito Federal, totalizando mais de 730 mil procedimentos especializados executados durante a período de março de 2015 a abril de 2016. Além disso, foi observado que cerca de $86 \%$ das metas foram atingidas pelos municípios. No entanto, algumas capitais apresentaram dificuldades no cumprimento de algumas metas, em especial na área de endodontia.
Embora o programa Brasil Sorridente seja uma iniciativa recente, é importante destacar seus avanços na atenção básica e também na especializada, principalmente a partir da incorporação dos LRPDs e dos $\mathrm{CEOs}^{1}$. Dessa forma, o presente estudo observou uma considerável quantidade de CEOs (59) localizados em 19 capitais brasileiras e no Distrito Federal, demonstrando uma significativa abrangência da atenção especializada em quase todas as capitais brasileiras. Além disso, a maioria dos CEOs observados era do tipo II, o que vai ao encontro dos achados de Goes et al. ${ }^{10}$ (2012), que observaram que cerca de 58\% dos CEOs brasileiros eram do tipo II. 
Em contrapartida, no presente estudo, foi observada uma maior quantidade de CEOs tipo III (27\%) comparando-se ao restante do país, que apresentou apenas cerca de $7 \%{ }^{10}$. Um estudo recente, avaliando os CEOs presentes no estado do Rio Grande do Sul, não observou a presença de CEOs tipo III no estado, além disso, cerca de $88 \%$ eram do tipo $\mathrm{I}^{8}$. $\mathrm{O}$ mesmo estudo também observou que os CEOs estavam distribuídos em cidades de grande porte populacional ${ }^{8}$. Este resultado talvez explique a elevada quantidade de CEOs instalados nas capitais brasileiras, uma vez que estas são, de forma geral, referências no atendimento de saúde.

Assim, o estabelecimento de CEOs pode ser favorecido nas capitais brasileiras devido ao fato de elas já serem centros: políticos, concentrando a maior parte legislativa e judiciária dos estados; educacionais, apresentando diversas universidades; e de saúde, concentrando grande parte dos hospitais e serviços de saúde. No entanto, é importante salientar que a iniciativa para a instalação dos CEOs é basicamente local, sendo um acordo exclusivo de municípios e estados, não sendo decisão da União a localização dos CEOs.

Uma grande quantidade de procedimentos especializados foi realizada nas capitais e no Distrito Federal durante o período avaliado. Cerca de 730 mil procedimentos, sem custo nenhum aos usuários do SUS, foram executados. Isso representa mais de 374 mil procedimentos periodontais, 242 mil procedimentos cirúrgicos e 113 mil procedimentos endodônticos, realizados de forma gratuita pelo SUS durante um único ano. Esses dados reforçam a importância da manutenção de políticas públicas como o Brasil Sorridente, principalmente em tempos de instabilidade política e econômica, em que diversos cortes na saúde vêm sendo realizados pelo governo federal.

Dessa forma, é importante destacar os avanços que a saúde bucal, incorporada na ESF, vem apresentando, de forma a dar embasamento ao papel desempenhado e destacar a importância de sua manutenção no contexto brasileiro ${ }^{11}$. Esses resultados tornam o Brasil um dos únicos países no mundo a oferecer um sistema de tratamento odontológico de forma gratuita e com a capacidade de ofertar uma expressiva quantidade de tratamentos odontológicos à população $0^{1}$.

Alguns estudos têm associado a produção odontológica com a quantidade de ESBs e a taxa de dentistas nos municípios, o que pode influenciar a organização dos sistemas de saúde e, consequentemente, a oferta de serviços à populaçãa $0^{7,12}$. Sabe-se que populações com status socioeconômico desfavorável tendem a ter condições de saúde mais vulneráveis, o que se reflete em incremento de cárie ${ }^{13,14}$ e de perda dental ${ }^{15}$. Regiões com pior índice de desenvolvimento humano parecem estar relacionadas com piores serviços na atenção secundária, da mesma forma como a cobertura pela ESB e o tempo de implementação dos serviços podem ser fatores importantes para o desempenho dos serviços em saú$\mathrm{de}^{16}$. Essas populações pertencem a grupos de risco que necessitam também de ações básicas de saúde direcionadas principalmente à prevenção. Além disso, é importante que o SUS disponibilize serviços de atenção especializada, para complementar as atividades que não podem ser desempenhadas nas $\mathrm{UBS}^{16}$.

Embora o presente estudo tenha observado uma expressiva quantidade de procedimentos realizados nas capitais com CEOs, é importante salientar que o acolhimento e o tratamento humanizado também são prioridades do Programa Nacional de Saúde Bucal ${ }^{1}$. Em relação à satisfação dos usuários de CEOs, um estudo realizado por Kitamura et al. ${ }^{17}$ (2016) observou que cerca de $87 \%$ dos usuários de CEOs, em uma macrorregião do estado de Minas Gerais, estavam satisfeitos com o serviço oferecido, e $62 \%$ dos indivíduos avaliaram o atendimento pelos cirurgiões-dentistas como excelente. A variável satisfação com o serviço esteve associada no modelo ajustado aos pacientes que apresentavam uma positiva autopercepção de saúde bucal e àqueles usuários que apresentaram um menor tempo de espera no atendimento ${ }^{17}$. De forma geral, a maior parte desses usuários foi referenciada a partir de UBS, demonstrando um indício do correto funcionamento do sistema de referência e contrarreferência. No entanto, um elevado tempo médio (63 dias) foi observado entre a referência na UBS e 0 atendimento dos usuários.

Apesar da considerável produção especializada bruta observada no presente estudo, é possível afirmar que algumas capitais apresentaram considerável dificuldade no cumprimento das metas, principalmente em relação à procedimentos de endodontia. Uma das capitais avaliadas cumpriu apenas 33\% das metas totais estabelecidas para a quantidade de CEOs cadastrados no CNES; além disso, nenhuma meta foi cumprida por este município para procedimentos de endodontia, o que pode acarretar um déficit expressivo de oferta de tratamentos para essa população. De forma semelhante, é possível observar que outras duas capitais também não cumpriram nenhuma das metas para procedimentos de endodontia. Esses resultados são preocupantes, tendo em vista a grande demanda de tratamentos endodônticos, sendo que a não oferta desses serviços pode induzir o usuário a optar - por falta de alternativa - pela exodontia. Além de ferir a autonomia na decisão do tratamento, a falta de oferta de tratamentos endodônticos agrava ainda mais a saúde bucal do usuário, que opta, assim, pela exodontia. Dessa forma, esse usuário necessitará de tratamento protético para reabilitação de sua saúde oral ${ }^{18}$. Resultados semelhantes foram observados nos CEOs do Rio Grande do Sul, onde foi observada uma grande dificuldade no cumprimento das metas, principalmente na área de endodontia ${ }^{8}$. 
De forma geral, o presente estudo observou um maior cumprimento das metas das capitais brasileiras e do Distrito Federal em comparação com os municípios do Rio Grande do Sul estudados por Pires et al. ${ }^{8}$ (2015). Quando considerado o cumprimento global das metas, o presente estudo observou que cerca de $86 \%$ delas foram atingidas. A área de endodontia - que apresentou o menor cumprimento - cumpriu 76\% das metas. É importante ressaltar que o presente estudo não considerou a análise dos procedimentos básicos que devem ser realizados em pacientes com necessidades especiais pelos CEOs.

Uma das grandes limitações deste estudo é a impossibilidade de separar os procedimentos realizados nos CEOs dos procedimentos realizados nas demais unidades de atendimento dos municípios. O SIASUS é um portal que oferece, de forma livre, os procedimentos realizados pelo SUS no Brasil. No entanto, a busca só é possível em nível municipal, não sendo possível averiguar a origem exata do procedimento. Isso restringe análises mais profundas sobre CEOs específicos e sobre os resultados encontrados. Devido a isso, os procedimentos básicos que os CEOs realizam em pacientes com necessidades especiais (que apresentam os mesmos códigos dos realizados nas UBS para os demais pacientes) foram excluídos da análise. No entanto, procedimentos especializados executados nas UBS podem ter sido computadas nos dados dos CEOS, sobre-estimando os resultados. Apesar de as UBS não serem destinadas à realização de procedimentos de média complexidade, essas unidades não apresentam, na maioria das vezes, estrutura para a realização de procedimentos especializados.

Caso alguma unidade básica das capitais avaliadas neste estudo tenha realizado procedimentos especializados, esses foram computados aos CEOs. Dessa forma, os dados apresentados neste estudo devem ser interpretados com cautela e talvez o real cumprimento das metas possa ser menor do que o observado. Isso torna ainda mais importante a averiguação do não cumprimento das metas por algumas capitais, principalmente em procedimentos de endodontia, que apresentaram considerável déficit - mesmo com a provável sobre-estimação.

Os gestores devem se manter atentos e averiguar o baixo cumprimento dessas metas nos municípios, uma vez que a portaria normatizadora prevê o acompanhamento e o monitoramento dos CEOs pelo mesmo sistema utilizado no presente estudo ${ }^{9}$. Além disso, estudos de heterocontrole devem ser realizados para prover um maior controle dos sistemas de saúde. Cabe, assim, ao CEO informar a quantidade de procedimentos no SIASUS, correndo o risco de sanções e até mesmo de descredenciamento, quando apresentar produção inferior ao estipulado. Assim, gestores devem monitorar os CEOs e realizar ações que favoreçam o cumprimento das metas.

\section{Conclusão}

O presente estudo observou uma elevada produção de média complexidade nas capitais brasileiras e no Distrito Federal, sendo que $86 \%$ das metas foram cumpridas, levando-se em consideração a quantidade de CEOs cadastrados no CNES. No entanto, algumas capitais apresentaram dificuldades no cumprimento das metas, principalmente na área de endodontia. Ressalta-se, assim, a importância da realização de estudos de heterocontrole e do monitoramento dos gestores quanto aos CEOs, a fim de criar ações que favoreçam o cumprimento das metas em locais com produção inferior à esperada.

\section{Abstract}

Dental Specialty Centers (Centros de Especialidades Odontológicas - CEOs) are specialized health facilities that should perform a minimum number of procedures. Objectives: this study aimed to describe the specialized dental production and report the achievement of goals in Brazilian capitals with CEOs. Materials and method: a retrospective longitudinal study was performed with a search for the CEOs listed in the National Registry of Health Establishments. The dental production was searched in the Outpatient Information System of the Brazilian Unified Health System for the period from May 2015 to April 2016. Results: fifty-nine CEOs were found and considered eligible for the present study. They were located in 19 Brazilian capitals and in the Federal District, whereas $48 \%$ were CEOs Type II. Approximately 730 thousand specialized procedures were performed during the 12 months evaluated. A rate of $86 \%$ of goals was met and surgery presented the highest achievement $(92 \%)$, followed by periodontics $(89 \%)$ and endodontics $(76 \%)$. One of the capitals achieved only $33 \%$ of the goals. Three capitals did not achieve any of the goals set for endodontic procedures. Conclusion: there was a great variation in the achievement of goals among capitals with CEOs. While some capitals showed high achievement of goals, others presented concerning data, especially for endodontic procedures.

Keywords: Health care assessment. Dental specialties. Oral health. Public health. Oral health care.

\section{Referências}

1. Pucca GA Jr., Gabriel M, de Araujo ME, de Almeida FC. Ten years of a National Oral Health Policy in Brazil: innovation, boldness, and numerous challenges. J Dent Res 2015; 94(10):1333-7.

2. Machado CV, Baptista TW, Nogueira C de O. Health policies in Brazil in the 2000s: the national priority agenda. Cad Saude Publica 2011; 27(3):521-32.

3. Brasil. Ministério da Saúde. Fundação Nacional de Saúde. Programa de Saúde da Família. Saúde dentro de Casa. Brasília, DF: Ministério da Saúde; 1994.

4. Soares CL. Constructing public oral health policies in Brazil: issues for reflection. Braz Oral Res. 2012; 26(Suppl 1):94102. 
5. Nickel DA, Lima FG, Bidigaray da Silva B. Dental care models in Brazil. Cad Saude Publica. 2008; 24(2):241-6.

6. Paim J, Travassos C, Almeida C, Bahia L, Macinko J. The Brazilian health system: history, advances, and challenges. Lancet 2011; 377(9779):1778-97.

7. Correa GT, Celeste RK. Association between coverage by oral health teams in the family health and the increase in dental care output in Brazilian municipalities, 1999 and 2011. Cad Saude Publica 2015; 31(12):2588-98.

8. Pires ALC, Gruendemann JLAL, Figueiredo GS, Conde MCM, Corrêa MB, Chisini LA. Secondary oral health care in the state of Rio Grande do Sul: descriptive analysis of the specialized production in cities with Dental Specialty Centers from the Outpatient Information System of the Unified Health System. RFO UPF 2015; 20(3):325-33.

9. Brasil. Ministério da Saúde. Portaria no 1.464 , de 24 de junho de 2011. Altera o Anexo da Portaria no 600/GM/MS, de 23 de março de 2006, que institui o financiamento dos Centros de Especialidades Odontológicas (CEO). Brasília, DF: Diário Oficial União; 2011.

10. Goes PS, Figueiredo N, Neves JC, Silveira FM, Costa JF, Pucca Junior GA, et al. Evaluation of secondary care in oral health: a study of specialty clinics in Brazil. Cad Saude Publica 2012; 28(Suppl):s81-9.

11. Scherer CI, Scherer MD. Advances and challenges in oral health after a decade of the "Smiling Brazil" Program. Rev Saude Publica 2015; 49.

12. Celeste RK, Moura FR, Santos CP, Tovo MF. Analysis of outpatient care in Brazilian municipalities with and without specialized dental clinics, 2010. Cad Saude Publica 2014; 30(3):511-21

13. Freire M do C, Reis SC, Figueiredo N, Peres KG, Moreira R da S, Antunes JL. Individual and contextual determinants of dental caries in Brazilian 12-year-olds in 2010. Rev Saude Publica 2013; 47(Suppl 3):40-9.
14. Hugo FN, Vale GC, Ccahuana-Vasquez RA, Cypriano S, de Sousa M da L. Polarization of dental caries among individuals aged 15 to 18 years. J Appl Oral Sci 2007; 15(4):253-8.

15. Seerig LM, Nascimento GG, Peres MA, Horta BL, Demarco FF. Tooth loss in adults and income: Systematic review and meta-analysis. J Dent 2015; 43(9):1051-9.

16. Cortellazzi KL, Balbino EC, Guerra LM, Vazquez F de L, Bulgareli JV, Ambrosano GM, et al. Variables associated with the performance of Centers for Dental Specialties in Brazil. Rev Bras Epidemiol 2014; 17(4):978-88.

17. Kitamura ES, Bastos RR, Palma PV, Leite ICG. Patient satisfaction evaluation at the Specialized Dental Centers in the Southeast Macro-region of Minas Gerais, Brazil, 2013. Epidemiol Serv Saúde 2016; 25(1):137-48.

18. Artigas PID, Oliveira EA, Júnior FBP, Sardinha SC, Andrade MGS. Investigação de fatores que limitam a autonomia de pacientes do SUS no processo de decisão de sua integridade bucal. R Ci Méd biol 2007; 6(3):316-23.

\section{Endereço para correspondência:}

Luiz Alexandre Chisini

Universidade do Vale do Taquari, Centro de

Ciências Biológicas e da Saúde

Rua Avelino Talini, 171

95914-014, Lajeado, Rio Grande do Sul, Brasil

Telefone: (53) 981121141

E-mail: alexandrechisini@gmail.com

Recebido: 07/06/18. Aceito: 24/07/18. 